\title{
Tropical Limit in Statistical Physics
}

\author{
M. Angelelli and B. Konopelchenko, \\ Department of Mathematics and Physics "Ennio De Giorgi", \\ University of Salento and sezione INFN, \\ Lecce, 73100, Italy.
}

\begin{abstract}
Tropical limit for macroscopic systems in equilibrium defined as the formal limit of Boltzmann constant $k \rightarrow 0$ is discussed. It is shown that such tropical limit is welladapted to analyse properties of systems with highly degenerated energy levels, particularly of frustrated systems like spin ice and spin glasses. Tropical free energy $F_{t r}(T)$ is a piecewise linear function of temperature $T$, tropical entropy is a piecewise constant function and the system has energy for which tropical Gibbs' probability has maximum. Properties of systems in the points of jump of entropy are studied. Systems with finite and infinitely many energy levels and phenomena of limiting temperatures are discussed.
\end{abstract}

\section{Introduction}

Singular (nonanalytic) limits of various types have shown up many times in physics and mathematics. Maslov's dequantization [1] 3], ultra-discrete integrable systems [4] 8 and tropical geometry $9 \sqrt{9}$ are three apparently disconnected fields where such a limit was most actively studied during last twenty years. Nowadays all of them are viewed as the different faces of the so-called tropical mathematics (see e.g. 14 16]). Tropical limit is characterized by a highly singular limiting behavior of the type $x=\exp \left(\frac{x_{t r}}{\varepsilon}\right)$ as the parameter $\varepsilon \rightarrow 0$. Elements $x_{t r}$ form an idempotent semiring with the tropical addition $\oplus$ and multiplication $\odot$ defined by $x_{1 t r} \oplus x_{2 t r}=\lim _{\varepsilon \rightarrow 0}\left(\varepsilon \ln \left(\exp \frac{x_{1 t r}}{\varepsilon}+\exp \frac{x_{2 t r}}{\varepsilon}\right)\right)=$ $\max \left\{x_{1 t r}, x_{2 t r}\right\}$ and $x_{1 t r} \odot x_{2 t r}=\lim _{\varepsilon \rightarrow 0}\left(\varepsilon \ln \left(\exp \frac{x_{1 t r}}{\varepsilon} \cdot \exp \frac{x_{2 t r}}{\varepsilon}\right)\right)=x_{1 t r}+x_{2 t r} 916$.

It was already noted in [13, 17,21$]$ that statistical physics seems to be the part of physics most naturally adapted to consider the tropical limit. Indeed, free energy $F$ of the macroscopic system in equilibrium is given by the formula 22$]$

$$
F=-k T \ln \sum_{n} g_{n} \exp \left(-\frac{E_{n}}{k T}\right)
$$


where $k$ is the Boltzmann constant, $T$ is the absolute temperature, $\left\{E_{n}\right\}$ is the energy spectrum of the system, $g_{n}$ are statistical weights (degeneracies) of the corresponding levels $E_{n}$ and the sum is performed over different energy levels. Thus, in the limit $k T \rightarrow 0$ one has the tropical sum in the r.h.s. of the formula (1.1) and $E_{n}$ and $F(k T \rightarrow 0)$ become elements of idempotent semiring refered in [21] as the thermodynamic semiring. In the papers 13,19 21] the tropical limit was identified with the limit $T \rightarrow 0$. With such a choice tropical free energy is equal to $E_{\min }$ and entropy $S_{t r}=0$ for the systems with finite $g_{n}$.

In this paper we argue that the formal limit $k \rightarrow 0$ is more appropriate avatar of tropical limit in statistical physics. At first glance the separation of $k$ and $T$ seems to be artificial and irrelevant since the r.h.s. of 1.1) and Gibbs' distribution

$$
w_{n}=\frac{\exp \left(-\frac{E_{n}}{k T}\right)}{\sum_{m} g_{m} \exp \left(-\frac{E_{m}}{k T}\right)}
$$

contain only the product $k T$. It is indeed so for systems with finite $g_{n}$.

An observation is that there exists a wide class of systems with exponentially large degeneracies $g_{n}$ for which the situation is quite different. At 1935 L. Pauling [23] showed that the degeneracy of the ground state of the ice is given by $g_{0}=\exp \left(N \ln \frac{3}{2}\right)$, where $N$ is the number of molecules. So the ice has (residual) entropy $S_{0}=k N \ln \frac{3}{2}$ at $T=0$ that is in excellent agreement with experimental data 24]. Several other systems like spin ices and spin glasses have exponentially large degeneracies of ground and excited states of the type $g_{n}=\exp \left(a_{n} N\right)$ with certain constants $a_{n}$ (see e.g. 22, 25 33]). In the thermodynamic limit $N \rightarrow \infty$ such $g_{n}$ have typical tropical behavior. A natural way to formalize this limit is to represent exponentially large degeneracies as $g_{n}=\exp \frac{S_{n}}{k}$ with finite $S_{n}$ and $k \rightarrow 0$. Physically it corresponds to the limit $N \rightarrow \infty, k \rightarrow 0$ with $k \cdot N=$ constant (gas constant $R$ ) and $S_{n}=a_{n} R$.

Thus, representing the degeneracies $g_{n}$ as $g_{n}=\exp \frac{S_{n}}{k}$ and defining $F_{t r}=\lim _{k \rightarrow 0} F$, one has at $T>0$

$$
F_{t r}(T)=-T \sum_{n} \oplus\left(-\frac{F_{n}}{T}\right)=\min \left\{F_{1}, F_{2}, . ., F_{n}, . .\right\}
$$

where $F_{n}=E_{n}-T S_{n}$ is a "microscopic" free energy associated with the energy level $E_{n}$. So $F_{t r}(T)$ is a piecewise linear function of temperature $T$. This leads to various consequences. For instance, the tropical entropy $S_{t r}=-\frac{\partial F_{t r}}{\partial T}=S_{n_{m i n}}$ where $n_{\text {min }}$ is the index of minimal free energy $F_{n_{\text {min }}}$ at temperature $T$ in the case when the minimum is attained only once. So $S_{t r}$ is a piecewise constant function of $T$. The value $S_{t r}(T=0)$ is the residual entropy of the macroscopic system at $T=0$. At certain singular values 
of $T S_{t r}$ exhibits jumps (entropy drop). Depending on the system it happens either at positive or negative temperatures.

These properties of the tropical limit $k \rightarrow 0$ trace quite well certain characteristic features of various frustrated systems similar to spin ices and spin glasses. In constrast these properties get lost in the limit $T \rightarrow 0$.

This is the main evidence in favour of the definition of the tropical limit as $k \rightarrow 0$. The second reason is that in such a limit the basic thermodynamic equations, like the first law $d E=T d S-p d V$ and relations between thermodynamic potentials, remain unaltered leaving temperature $T$ to be a free positive or negative parameter. In addition the limit $k \rightarrow 0$ resembles very much that of $\hbar \rightarrow 0$ in Maslov's dequantization.

Tropical limit of Gibbs' distribution 1.2 has rather interesting properties too. Tropical probability $w_{n, t r}=\lim _{k \rightarrow 0}\left(k \cdot \ln w_{n}\right)$ takes values in the interval $(-\infty, 0]$ and is equal to

$$
w_{n, t r}=-S_{n}+\frac{F_{t r}-F_{n}}{T}
$$

The tropical probability $W_{n, t r}$ for the system to have energy $E_{n}$ is

$$
W_{n, t r}=w_{n, t r}+S_{n}=\frac{F_{t r}-F_{n}}{T}
$$

and it is normalized by the condition $\sum_{n} \oplus W_{n, t r}=0$.

These tropical Gibbs' distributions describe fine structure of the states with exponentially small usual probabilities $w_{n} \sim \exp \left(-\frac{S_{n}}{k}\right)$. It is shown that tropical probabilities and entropy have a peculiar behavior at the singular values $T^{*}$ of temperature at which jump of $S_{t r}$ is observed.

Systems with finitely many energy levels are considered as illustrative examples. Tropical limit of the systems with infinite number of energy levels, the phenomenon of limiting temperatures and existence of intervals of forbidden temperatures are discussed too.

It is noted that the limit $k \rightarrow 0$ viewed as the limit of vanishing white noise for systems with finite degeneracies has been discussed in a different context in 34].

The paper is organized as follows. In section 2 general definitions and formulas are presented. Singularities appearing in tropical limit are analysed in next section 3. Systems with finite number of energy levels are considered in section 4 . In section 5 the systems with infinitely many energy levels bounded and unbounded from below and the existence of limiting temperatures are discussed. 


\section{Tropical Gibbs' distribution and free energy}

So we will consider macroscopic systems in equilibrium and will study their limiting behavior as (formally) $k \rightarrow 0$. Introducting the energy level "entropy" $S_{n}=k \ln g_{n}$ and assuming that $S_{n}$ are finite, one has the following form of partition function

$$
Z=\sum_{n \geq 1} \exp \left[\frac{1}{k}\left(S_{n}-\frac{E_{n}}{T}\right)\right]=\sum_{n \geq 1} \exp \left(-\frac{F_{n}}{k T}\right)
$$

where $F_{n} \equiv E_{n}-T S_{n}$ is the "energy-level" free energy and energies $E_{n}$ are ordered as $0<E_{1}<E_{2}<\ldots$. One observes that the degeneracies $g_{n}=\exp \frac{S_{n}}{k}$ with finite $S_{n}>0$ and Boltzmann weights $\exp \left(-\frac{E_{n}}{k T}\right)$ behave quite differently as $k \rightarrow 0$. So in the tropical limit we will have sort of Bergmann's logarithmic limit set 35].

Tropical limit of probability $w_{n}$, in general, is naturally associated with its singular behavior of the form $w_{n}=\tilde{w}_{n} \cdot \exp \frac{w_{n, t r}}{\varepsilon}$ with small positive parameter $\varepsilon, 0<\tilde{w}_{n} \leq 1$ and $w_{n, t r}=\lim _{\varepsilon \rightarrow 0}\left(\varepsilon \ln w_{n}\right)$. Tropical probability $w_{n, t r}$ varies in the interval $(-\infty, 0]$. The interval $0<w_{n} \leq 1$ collapses into $\{0\}$ while exponentially small usual probabilities $w_{n}$ are represented by the whole semi-line $(-\infty, 0)$ for $w_{n, t r}$ and numbers $\tilde{w}_{n}$. The meaning of the quantities $w_{n, t r}$ and $\hat{w}_{n}$ is clarified by the formula $\ln w_{n}=\frac{w_{n, t r}}{\varepsilon}+\ln \tilde{w}_{n}+\ldots$. So singular behavior under consideration is characterized by a simple pole behavior of $\ln w_{n}$ as a function of the small parameter $\varepsilon$ : $w_{n, t r}$ is the residue at this pole while $\ln \tilde{w}_{n, t r}$ is the first regular nondominant term. In generic regular case it is sufficient to consider the dominant pole term and, hence, the tropical probability $w_{n, t r}$. Contribution of nondominant term $\ln \tilde{w}_{n}$ becomes crucial, as we shall see, in the singular situations when limit $\varepsilon \rightarrow 0$ ceases to be uniquely defined.

Under the assumption that all $F_{n}$ are distinct the tropical limit of Gibbs' probabilities 1.2 is given by $(\varepsilon=k)$

$$
w_{n, t r}=-\frac{E_{n}}{T}-\max \left\{-\frac{F_{1}}{T},-\frac{F_{2}}{T}, \ldots\right\}=-S_{n}-\frac{F_{n}}{T}+\min \left\{\frac{F_{1}}{T}, \frac{F_{2}}{T}, \ldots\right\}
$$

$\operatorname{Denoting}\left(\frac{F}{T}\right)_{\min }:=\min \left\{\frac{F_{1}}{T}, \frac{F_{2}}{T}, \ldots\right\}$, one gets

$$
w_{n, t r}=-S_{n}-\frac{F_{n}}{T}+\left(\frac{F}{T}\right)_{\text {min }} .
$$

Normalization condition for these tropical probabilities is the limit $k \rightarrow 0$ of the condition $\sum_{n} g_{n} \cdot w_{n}=1$ and it is given by

$$
\sum_{n} \oplus\left(S_{n}+w_{n, t r}\right)=0
$$


In particular, for $n=n_{0}$ such that $\frac{F_{n_{0}}}{T}=\left(\frac{F}{T}\right)_{\min }$, one has

$$
w_{n_{0}, t r}=-S_{n_{0}} .
$$

So, the entropies $S_{n}$ are, in fact, the tropical Gibbs' probabilities to find the system in certain state with energy $E_{n}$. Probability $W_{n}$ for the system to have energy $E_{n}$ at small $k$ and $T>0$ is equal to $W_{n}=g_{n} \exp \frac{w_{n, t r}}{k}=\exp \frac{W_{n, t r}}{k}$ and, hence, tropical probability $W_{n, t r}$ for the system to have energy $E_{n}$ is equal to

$$
W_{n, t r}=\frac{F_{t r}-F_{n}}{T}
$$

These tropical probabilities obey the normalization condition $\sum_{n} \oplus W_{n, t r}=\max \left\{W_{n, t r}\right\}=$ 0 . Also in the limit $k \rightarrow 0$ for usual probabilities one gets $W_{n_{0}}^{n}=1$ and $W_{n \neq n_{0}}=0$ and the tropical energy $E_{t r}$ of the system is

$$
E_{t r}=\lim _{k \rightarrow 0}\left(\sum_{n \geq 1} W_{n} E_{n}\right)=E_{n_{0}} .
$$

The tropical Gibbs' distribution provides us with the fine description of the energy levels.

Tropical limit of the free energy 1.1 is given by

$$
F_{t r}(T)=-T \max \left\{-\frac{F_{1}}{T},-\frac{F_{2}}{T}, \ldots\right\}=T \min \left\{\frac{F_{1}}{T}, \frac{F_{2}}{T}, \ldots\right\}
$$

or

$$
-\frac{F_{t r}(T)}{T}=-\sum_{n \geq 1} \oplus\left\{-\frac{F_{n}}{T}\right\}
$$

assuming that $\max \left\{-\frac{F_{n}}{T}, n \geq 1\right\}$ exists. Thus, the tropical free energy is the additive tropical sum of the free energies $F_{n}$ of energy levels.

Tropical free energy $F_{t r}$ is, in general, a piecewise linear function of temperature $T$. For instance, for positive $T$ it is

$$
F_{t r}=\min \left\{E_{1}-T S_{1}, E_{2}-T S_{2}, \ldots\right\}
$$

In this case the tropical limit $S_{t r}$ of the entropy defined by the standard formula $S_{t r}=$ $-\frac{\partial F_{t r}}{\partial T}$ is equal to $S_{t r}=S_{n_{0}}$ where $F_{n_{0}}=F_{m i n}$. With such a definition of $F_{t r}$ and $S_{t r}$ one has

$$
d F_{t r}=-S_{t r} d T-p_{t r} d V
$$


where $p_{t r}=-\left(\frac{\partial F_{t r}}{\partial V}\right)_{T}$ and $F_{t r}=E_{t r}-T S_{t r}$.

The same tropical entropy is obtained as the limit $k \rightarrow 0$ of the standard formula $S=-k \overline{\ln w_{n}}$. Indeed, at $T>0$ the Gibbs' probabilities are

$$
w_{n, t r}=-S_{n}+\frac{F_{t r}-F_{n}}{T}
$$

and

$$
w_{n_{0}, t r}=-S_{t r}
$$

Using these formulas, one gets

$$
\begin{gathered}
-\lim _{k \rightarrow 0}\left(k \overline{\ln w_{n}}\right)=-\lim _{k \rightarrow 0}\left(k \sum_{n \geq 1} g_{n} w_{n} \ln w_{n}\right)= \\
=-\lim _{k \rightarrow 0}\left(k \sum_{n \geq 1} \exp \left(\frac{S_{n}}{k}\right) \cdot\left[\exp \left(\frac{w_{n, t r}}{k}+\mathcal{O}(k)\right)\right] \cdot\left[-\frac{S_{n}}{k}-\frac{F_{n}-F_{t r}}{k T}+\mathcal{O}(k)\right]\right)= \\
=S_{n_{0}}=S_{t r} .
\end{gathered}
$$

Tropical entropy is a piecewise constant function of temperature $T$. At the limit $T \rightarrow 0$ one has $S_{t r}=S_{n_{0}}(T \rightarrow+0)=S_{1}$. So $S_{1}$ is the residual entropy of the system at absolute zero. Tropical entropy $S_{t r}$ is a constant and hence the specific heat $C_{V, t r}=$ $T\left(\frac{\partial S_{t r}}{\partial T}\right)_{V}=0$. At the limit $T \rightarrow-0, S_{t r}=S_{n^{*}}$, where $S_{n^{*}}$ is the entropy of the level with largest energy.

Formulae 2.4 2.5, 2.6 have a simple probabilistic interpretation. Indeed, the tropical probability $W_{n, t r}$ for the system to have energy $E_{n}$ is given by (2.6) where $F_{t r}=F_{\min }=$ $F_{n_{0}}$. Thus, the system at temperature $T(>0)$ has such energy $E_{n}$ for which the tropical probability $W_{n, t r}$ is maximal, i.e. zero. This law of tropical probability maximum is a clear manifestation of the relevance of the limit $k \rightarrow 0$.

Finally, we note that in the case of all $S_{n}=0$ one has the tropical limit for the system with finite degeneracies for which $F_{t r}=E_{1}$.

\section{$3 \quad$ Singularity in the tropical limit}

The formulae and results presented in the previous section are valid in generic situation when all $F_{n}$ are distinct.

Singularity (nonuniqueness) of the tropical limit arises in the case when $F_{\min }$ is attained on two or more $F_{n}$ (see e.g. $9,10,12,13$ ). In such a situation the tropical free energy $F_{t r}$ is nondifferentiable as a function of temperature $T$ at certain $T=T^{*}$.

How other tropical quantities behave at these temperatures? Let $F_{n_{0}}$ and $F_{n_{0}+1}$ be two successive minima of $\left\{F_{n}\right\}$ with $S_{n_{0}} \neq S_{n_{0}+1}$. At $F_{n_{0}}=F_{m i n}$ tropical entropy is $S_{n_{0}}$. 
For $F_{\text {min }}=F_{n_{0}+1}$ one has $S_{t r}=S_{n_{0}+1}$.

Values of $F_{n_{0}}$ and $F_{n_{0}+1}$ coincide at the temperature $\left(F_{\min }\left(T^{*}\right)=F_{n_{0}}\left(T^{*}\right)=F_{n_{0}+1}\left(T^{*}\right)\right)$

$$
T^{*}=\frac{E_{n_{0}}-E_{n_{0}+1}}{S_{n_{0}}-S_{n_{0}+1}} .
$$

Sign of $T^{*}$ coincides with $\operatorname{sign}\left(S_{n_{0}+1}-S_{n_{0}}\right)$. If $S_{n_{0}+1}>S_{n_{0}}$ then $F_{n_{0}}<F_{n_{0}+1}$ at $T<T^{*}$ and $F_{n_{0}+1}<F_{n_{0}}$ at $T>T^{*}$. So at $T<T^{*}$ the tropical entropy is equal to $S_{n_{0}}$ while at $T>T^{*}$ one has $S_{t r}=S_{n_{0}+1}$. Thus, the tropical entropy jumps when the temperature $T$ passes the point $T^{*}$. This jump of entropy $S_{t r}$ at $T=T^{*}$ is the tropical trace of the entropy drop phenomenon discussed for spin glasses in $28,30,33$. Using the formula 2.6 for the difference of tropical probability $W_{n_{0}, t r}(T)$ and $W_{n_{0}+1, t r}(T)$, one gets

$$
\Delta W_{t r}=W_{n_{0}, t r}(T)-W_{n_{0}+1, t r}(T)=\frac{F_{n_{0}+1}-F_{n_{0}}}{T}=\Delta S_{t r} \cdot\left(\frac{T^{*}}{T}-1\right) .
$$

So $\Delta W_{t r}$ behaves as $\sim\left(T^{*}-T\right)$ near the singular temperature $T^{*}$.

In order to calculate probabilities and entropy at singular point $T=T^{*}$ one should take into account not only dominant terms but also first regular nondominant terms. Indeed in the case, where $F_{\min }$ is attained precisely on $F_{n_{0}}$ and $F_{n_{0}+1}$, from the Gibbs' distribution 1.2 at small $k$ one gets

$$
\ln w_{n}=-\frac{S_{n}}{k}-\frac{F_{n}\left(T^{*}\right)-F_{n_{0}}\left(T^{*}\right)}{k T^{*}}-\ln 2+\mathcal{O}(k) .
$$

In particular

$$
\begin{aligned}
\ln w_{n_{0}} & =-\frac{S_{n_{0}}}{S^{k}}-\ln 2+\mathcal{O}(k), \\
\ln w_{n_{0}+1} & =-\frac{S_{n_{0}+1}}{k}-\ln 2+\mathcal{O}(k), \\
W_{n_{0}, t r} & =W_{n_{0}+1, t r}=-k \cdot \ln 2 .
\end{aligned}
$$

Hence, at $T=T^{*}$ and small $k$

$$
\begin{aligned}
w_{n_{0}} & =\frac{1}{2} \exp \left(-\frac{S_{n_{0}}}{k}\right), \\
w_{n_{0}+1} & =\frac{1}{2} \exp \left(-\frac{S_{n_{0}+1}}{k}\right) .
\end{aligned}
$$

Using definition of tropical entropy, one readily obtains

$$
S_{t r}\left(T^{*}\right)=-\lim _{k \rightarrow 0}\left(k \overline{\ln w\left(T^{*}\right)}\right)=\frac{1}{2}\left(S_{n_{0}}+S_{n_{0}+1}\right) .
$$

One gets the same result by direct calculation, namely,

$S_{t r}\left(T^{*}\right)=-\lim _{k \rightarrow 0}\left(k \overline{\ln w\left(T^{*}\right)}\right)=$ 


$$
\begin{gathered}
=-\lim _{k \rightarrow 0}\left[k \frac{\exp \left(-\frac{F_{n_{0}}}{k T}\right)}{2 \exp \left(-\frac{F_{n_{0}}}{k T}\right)+\sum_{n \neq n_{0}, n_{0}+1} \exp \left(-\frac{F_{n}}{k T}\right)}\left(-\frac{S_{n_{0}}}{k}-\frac{S_{n_{0}+1}}{k}+\mathcal{O}\left(k^{0}\right)\right)\right]- \\
-\lim _{k \rightarrow 0}\left[k \sum_{m \neq n_{0}, n_{0}+1} \frac{\exp \frac{F_{n_{0}}-F_{m}}{k T}}{\left(2+\sum_{n \neq n_{0}, n_{0}+1} \exp \frac{F_{n_{0}}-F_{n}}{k T}\right)}\left(-\frac{S_{m}}{k}-\frac{F_{m}-F_{n_{0}}}{k T}+\mathcal{O}\left(k^{0}\right)\right)\right]= \\
=\frac{1}{2}\left(S_{n_{0}}+S_{n_{0}+1}\right) .
\end{gathered}
$$

Note that at $T=T^{*}$ the usual probabilities $W_{n_{0}}=g_{n_{0}} \cdot w_{n_{0}}$ and $W_{n_{0}+1}=g_{n_{0}+1}$. $w_{n_{0}+1}$ for the system to have energies $E_{n_{0}}$ and $E_{n_{0}+1}$, respectively, are equal $W_{n_{0}}=W_{n_{0}+1}$ $=\frac{1}{2}$. One also has $E_{t r}\left(T^{*}\right)=\frac{1}{2}\left(E_{n_{0}}+E_{n_{0}+1}\right)$. Note that in the generic case one has $\ln 1$ instead of $\ln 2$ in $(3.3)$ and, hence, the formula 2.13). If $F_{\min }$ would be attained on $m F_{n}$ 's, one would have $\ln w_{n_{0}}=-\frac{S_{n_{0}}}{k}-\ln m+\mathcal{O}(k)$.

In more details the importance of nondominant terms in singular points and their relevance to the deformation of idempotent semiring will be discussed elsewhere.

\section{Systems with finite number of energy levels}

We begin with the simplest non-trivial case of two level systems and $S_{2}>S_{1}$. For the systems with finite number of energy levels there is no constraint on the sign of temperature (see $[22]$ ). Thus the tropical free energy at $T>0$ is

$$
F_{t r}(T)=\min \left\{E_{1}-T S_{1}, E_{2}-T S_{2}\right\}
$$

and

$$
F_{t r}=\max \left\{E_{1}-S_{1} T, E_{2}-S_{2} T\right\}
$$

for $T<0$.

Transition temperature $T^{*}=\frac{E_{2}-E_{1}}{S_{2}-S_{1}}>0$. At $0<T<T^{*}$ one has

$$
F_{t r}(T)=E_{1}-S_{1} T
$$

and tropical energy and entropy are $E_{t r}=E_{1}$ and $S_{t r}=S_{1}$.

At $T>T^{*}$ and $T<0$

$$
F_{t r}(T)=E_{2}-S_{2} T
$$

and $E_{t r}=E_{2}, S_{t r}=S_{2}$. 
The graph of $F_{t r}(T)\left(\right.$ at $\left.\frac{E_{2}}{E_{1}}<\frac{S_{2}}{S_{1}}\right)$ is presented in figure 4.1 .

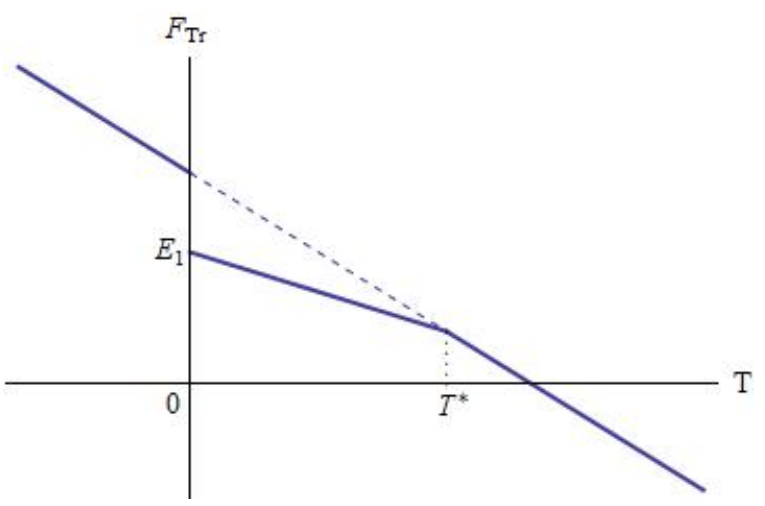

Figure 4.1: $F_{t r}$ in two level case, $S_{2}>S_{1}$.

The residual entropy is equal to $S_{t r}(T \rightarrow+0)=S_{1}$, while $S_{t r}(T \rightarrow-0)=S_{2}$. The jump of $S_{t r}$ at $T=T^{*}$ is $S_{2}-S_{1}$ and $S_{t r}\left(T^{*}\right)=\frac{1}{2}\left(S_{1}+S_{2}\right), E_{t r}\left(T^{*}\right)=\frac{1}{2}\left(E_{1}+E_{2}\right)$.

At $T<T^{*}$ the tropical probability $W_{1, t r}$ to have energy $E_{1}$ is equal to $W_{1, t r}=0$ while the probability $W_{2, t r}$ to have energy $E_{2}$ is $W_{2, t r}=\left(S_{2}-S_{1}\right) \cdot\left(1-\frac{T^{*}}{T}\right)<0$. At $T>T^{*}$ the situation is opposite, namely, $W_{2, t r}=0, W_{1, t r}=\left(S_{1}-S_{2}\right) \cdot\left(1-\frac{T^{*}}{T}\right)<0$.

In the case $S_{1}>S_{2}$ the transition temperature $T^{*}$ is negative and the graph of $F_{t r}(T)$ is given on the figure 4.2 .

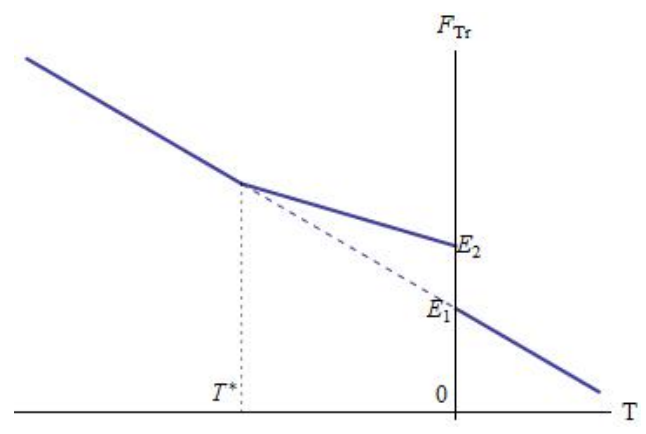

Figure 4.2: $F_{t r}$ in two level case, $S_{1}>S_{2}$.

So $E_{t r}(T \rightarrow+0)=E_{1}$ and $S_{t r}(T \rightarrow+0)=S_{1}$ again, $S_{t r}(T \rightarrow-0)=S_{2}$ and $S_{t r}\left(T^{*}\right)=\frac{1}{2}\left(S_{1}+S_{2}\right), E_{t r}\left(T^{*}\right)=\frac{1}{2}\left(E_{1}+E_{2}\right)$. Since negative temperatures are higher than positive one, in this case transition takes place in "higher" temperature.

Finally, if $S_{1}=S_{2}$, one has (see figure (4.3)) $T^{*}=+\infty, E_{t r}(T>0)=E_{1}, S_{t r}(T>$ $0)=S_{1}, E_{t r}(T<0)=E_{2}, S_{t r}(T<0)=S_{2}$.

For the system with three energy levels $E_{1}<E_{2}<E_{3}$ we will consider here only three characteristic cases.

1. $S_{1}<S_{2}<S_{3}$. At $T>0, F_{t r}(T)=\min \left\{E_{1}-S_{1} T, E_{2}-S_{2} T, E_{3}-S_{3} T\right\}$.

Singular (transition) values of $T$ are 


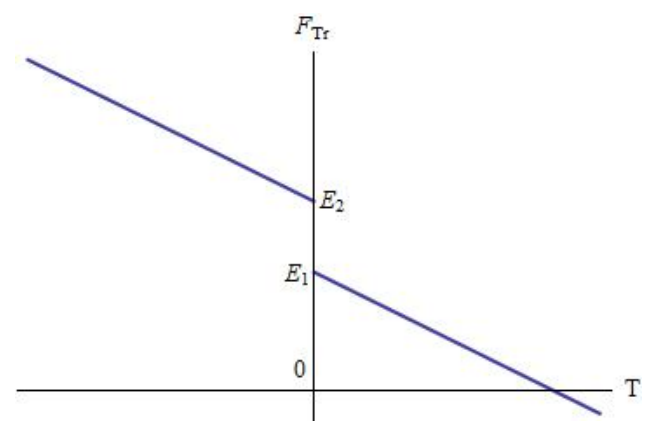

Figure 4.3: $F_{t r}$ in two level case, $S_{1}=S_{2}$.

$$
T_{i k}^{*}=\frac{E_{i}-E_{k}}{S_{i}-S_{k}}>0, \quad i, k=1,2,3, i \neq k .
$$

They are not independent since

$$
\left(S_{1}-S_{2}\right) T_{12}^{*}+\left(S_{2}-S_{3}\right) T_{23}^{*}+\left(S_{3}-S_{1}\right) T_{31}^{*}=0 .
$$

As the consequence, $T_{12}^{*}-T_{23}^{*}$ and $T_{13}^{*}-T_{23}^{*}$ have the same sign.

In the case $T_{23}^{*}>T_{12}^{*}$ and $T_{13}^{*}>T_{12}^{*}$ the graph of $F_{t r}$ is given on the figure (4.4)

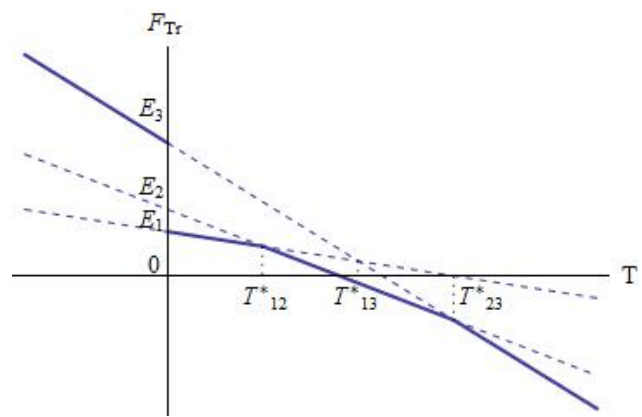

Figure 4.4: $F_{t r}$ in three level case, $S_{1}<S_{2}<S_{3}, T_{23}^{*}>T_{13}^{*}>T_{12}^{*}$.

So at $0<T<T_{12}^{*}, F_{t r}=E_{1}-S_{1} T, E_{t r}=E_{1}, S_{t r}=S_{1}$. For $T_{12}^{*}<T<T_{23}^{*}$ one has $F_{t r}=E_{2}-S_{2} T, E_{t r}=E_{2}, S_{t r}=S_{2}$; at $T>T_{23}^{*}$ and $T<0$ it's $F_{t r}=E_{3}-S_{3} T$, $E_{t r}=E_{3}, S_{t r}=S_{3}$.

2. If, instead, $T_{23}^{*}<T_{13}^{*}<T_{12}^{*}$ one has quite different behavior of the system (figure 4.5. So at $0<T<T_{13}^{*}, F_{t r}=E_{1}-S_{1} T, E_{t r}=E_{1}, S_{t r}=S_{1}$, while for $T>T_{13}^{*}$, $F_{t r}=E_{3}-S_{3} T, E_{t r}=E_{3}, S_{t r}=S_{3}$.

Thus depending on parameter $E_{i}, S_{i}$ one may have two singular values of temperature or only one. In the first case, with increase of temperature from $T=0$, the system in the tropical limit first jumps from the macroscopic state of the system 


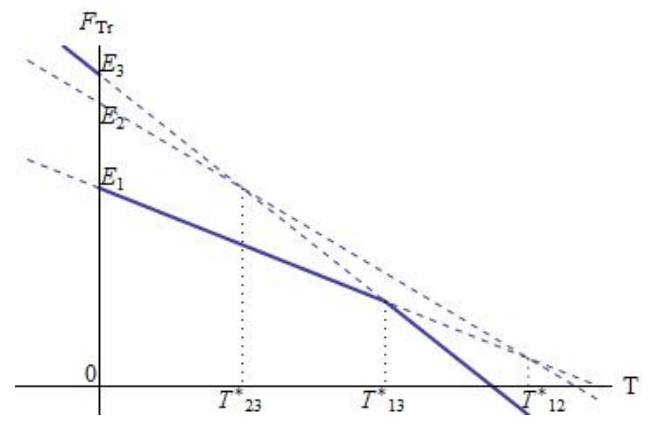

Figure 4.5: $F_{t r}$ in three level case, $S_{1}<S_{2}<S_{3}, T_{23}^{*}<T_{13}^{*}<T_{12}^{*}$.

with energy $E_{1}$ and entropy $S_{1}$ to the state with energy $E_{2}$ and entropy $S_{2}$ at temperature $T_{12}^{*}$, and then at temperature $T_{23}^{*}$ it jumps from $E_{2}$ and $S_{2}$ to $E_{3}$ and $S_{3}$ respectively.

In the second case, the system jumps only once at temperature $T_{13}^{*}$ from the state with $E_{1}, S_{1}$ to the state with $E_{3}, S_{3}$.

At $S_{1}>S_{2}>S_{3}$ one has similar behavior at negative $T$.

3. Our third example corresponds to $S_{3}<S_{1}<S_{2}$. In this case $T_{23}^{*}<0$ and $F_{t r}$ is given in figure 4.6.

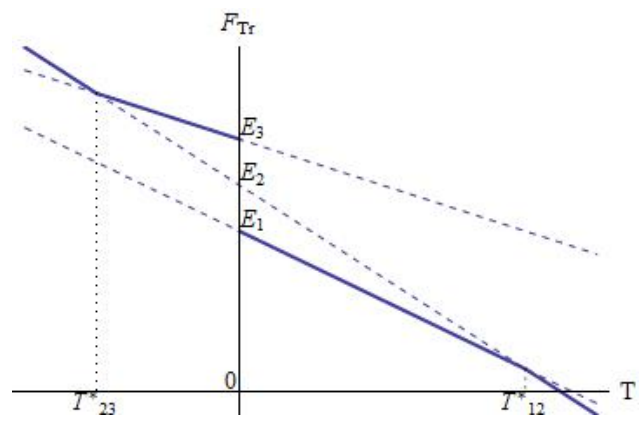

Figure 4.6: $F_{t r}$ in three level case, $S_{3}<S_{1}<S_{2}$.

So at $0<T<T_{12}^{*}, E_{t r}=E_{1}, S_{t r}=S_{1}$. At $T_{12}^{*}<T<+\infty$ one has $E_{t r}=E_{2}$, $S_{t r}=S_{2}$. At $-\infty<T<T_{23}^{*}, F_{t r}=E_{2}-S_{2} T, E_{t r}=E_{2}, S_{t r}=S_{2}$, while at $0>T>T_{23}^{*}, E_{t r}=E_{3}, S_{t r}=S_{3}$.

In all above three cases the system has at most two singular (transition) temperatures. One can show that the same is true in all other cases.

For the system with $n$ energy levels there is number of different cases. In principle there are $\frac{n \cdot(n-1)}{2}$ singular values of temperature $T_{i j}^{*}=\frac{E_{i}-E_{j}}{S_{i}-S_{j}}=T_{j i}^{*}$, for $i, j=$ $1, \ldots, n, i \neq j$. Due to the identities

$$
\left(S_{i}-S_{k}\right) T_{i k}^{*}+\left(S_{k}-S_{l}\right) T_{k l}^{*}+\left(S_{l}-S_{i}\right) T_{l i}^{*}=0, i \neq k \neq l \neq i, i, k, l=1, \ldots, n,
$$


there is a number of constraints on different $T_{i k}^{*}$. It is not difficult to show that the tropical free energy $F_{t r}(T)$ may have at most $n$ linear pieces and hence $n-1$ transition temperatures $T^{*}$. In all situations, $E_{t r}(T \rightarrow+0)=E_{1}, S_{t r}(T \rightarrow+0)=S_{1}$ and $E_{t r}(T \rightarrow$ $-0)=E_{n}, S_{t r}(T \rightarrow-0)=S_{n}$.

At $T>0$ the thermodynamical energy $E_{t r}$ of the system with $n$ energy levels may assume one, two, up to $n$ different values depending on relations between $S_{1}, S_{2}, \ldots, S_{n}$.

We would like to note that systems with finite number of energy levels appear also in the study of frustrated systems with different "micro-basins" of macroscopic systems characterized by energies $E_{n}$. For such type of systems the transition temperatures $T^{*}$ considered above represent the tropical limit of the points of first order phase transitions with latent heats $q_{i k}=T_{i k}^{*}\left(S_{i}-S_{k}\right)=E_{i}-E_{k}$.

\section{Systems with infinitely many energy levels and lim- iting temperatures}

Tropical limit for systems with countable number of energy levels is defined in the same manner as in the finite case. The tropical free energy $F_{t r}$ is an infinite tropical sum

$$
F_{t r}(T)=-T \sum_{n} \oplus\left(-\frac{F_{n}}{T}\right)
$$

viewed as the formal limit of the standard tropical sum when the number of terms goes to infinity.

For the systems with the energy spectrum bounded from below, the sum in 5.1 is performed over all $n \geq 1$, with $0<E_{1}<E_{2}<\ldots<E_{n}<\ldots$. If the energy spectrum of the system is unbounded from below, then the sum is over all integers $-\infty<n<\infty$ and energies ordered as $E_{n}<E_{n+1}$.

Existence or nonexistence of $F_{t r}(T)$ for certain temperatures, i.e. convergence or divergence of the infinite sum in (5.1), is the key novel feature of infinite case in comparison with the finite one. This is the tropical $k \rightarrow 0$ trace of the existence of limiting temperatures for usual macroscopic systems, i.e. existence of intervals of temperature for which system cannot stay in equilibrium (see $22,36,37$ ).

Let us begin with a system with the energy spectrum bounded from below and unbounded from above. At $T>0$ one has $F_{t r}(T)=-T \max \left\{-\frac{F_{1}}{T},-\frac{F_{2}}{T}, \ldots, \frac{F_{n}}{T}, \ldots\right\}=$ $\min \left\{F_{1}, F_{2}, F_{3}, \ldots\right\}$. In the case when the minimum of $\left\{F_{1}, F_{2}, \ldots\right\}$ exists, i.e. it is finite, one has the behavior which is just the limit of that considered in the previous section.

Singularity of $F_{t r}(T)$ may occur for such a temperature $T_{S}$ when, beginning from certain $F_{n_{0}}(T)$ all $F_{n_{0}+k}(T)>F_{n_{0}+k+1}(T)$ (at $T>T_{S}$ and $\left.k=0,1,2, \ldots\right)$ and, hence, $\min \left\{F_{1}, F_{2}, \ldots\right\}$ does not exist. It is achieved, for instance, in the case when $S_{n_{0}+k+1}>$ 
$S_{n_{0}+k}>0, \frac{E_{n_{0}+k+1}}{S_{n_{0}+k+1}} \leq \frac{E_{n_{0}+k}}{S_{n_{0}+k}}, k=0,1,2, \ldots$ and set of differencies $S_{n_{0}+k+1}-S_{n_{0}+k}$ has positive lower bound. Indeed, it is easy to see, that at $T>T_{S+}=\frac{E_{n_{0}}}{S_{n_{0}}}$ one has

$$
\begin{aligned}
& F_{n_{0}}(T)=S_{n_{0}}\left(T_{S+}-T\right)>F_{n_{0}+1}(T)=S_{n_{0}+1}\left(\frac{E_{n_{0}+1}}{S_{n_{0}+1}}-T\right)>\ldots \\
& \ldots F_{n_{0}+k}=S_{n_{0}+k}\left(\frac{E_{n_{0}+k}}{S_{n_{0}+k}}-T\right)>\ldots, k=2,3, \ldots .
\end{aligned}
$$

Thus, for $T>T_{S+}$, this sequence has no lower limit, so $\min \left\{F_{n}\right\}$ is not bounded from below, infinite tropical sum diverges and $F_{t r}(T)$ does not exist. So our system can be in equilibrium only for temperatures $T$ in the interval $0 \leq T<T_{S+}$. This phenomenon has a simple probabilistic interpretation similar to that of full nontropical case (see e.g. 36, 37]). Indeed, for "forbidden" values of temperature, $F_{t r}=F_{\min } \rightarrow-\infty$. Even if one works in $\mathbb{R}_{\max }=(\mathbb{R} \cup\{-\infty\}$, $\max ,+)$, and hence $-\infty \in \mathbb{R}_{\max }$, the formula 2.6 for $W_{n, t r}$ does not define any distribution of probabilities obeying the tropical normalization condition and the system cannot stay in equilibrium.

In the particular case $n_{0}=1$ and $\frac{E_{n}}{S_{n}}=a$, i.e. $S_{n}=\frac{E_{n}}{a}, n=1,2 \ldots, F_{n}=$ $E_{n}\left(1-\frac{T}{a}\right)$ the limiting temperature is $T_{S+}=a$. Such limiting temperature is (not surprisingly) the same as in the corresponding full nontropical case (see [37]). In the tropical limit the system with temperature $T<T_{S+}=\frac{E_{n_{0}}}{S_{k_{0}}}$ has the same properties as the system with $n_{0}$ energy levels, i.e. it may have at most $n_{0}$ values of energy and entropy. In particular, in the case $n_{0}=1$, e.g. $S_{n}=\frac{E_{n}}{a}$, the system has energy $E_{1}$ and entropy $S_{1}$ for all $T<T_{S+}$.

Different limits for temperature arise in the case when all $S_{n_{0}+k}<0, k=0,1,2, \ldots$ for some $n_{0}$. Indeed, if $S_{n_{0}+k+1}<S_{n_{0}+k}<0$, and $\frac{S_{n_{0}+k+1}}{E_{n_{0}+k+1}} \geq \frac{S_{n_{0}+k}}{E_{n_{0}+k}}, k=0,1,2, \ldots$, then at $\frac{E_{n_{0}}}{S_{n_{0}}}<T<0$ one has $-\frac{F_{n_{0}}}{T}=E_{n_{0}}\left(\frac{S_{n_{0}}}{E_{n_{0}}}-\frac{1}{T}\right)<-\frac{F_{n_{0}+1}}{T}=E_{n_{0}+1}\left(\frac{S_{n_{0}+1}}{E_{n_{0}+1}}-\frac{1}{T}\right)<\ldots$ $\ldots<-\frac{F_{n_{0}+k}}{T}<\ldots$. So, $\max \left\{-\frac{F_{n}}{T}\right\}$ does not exist and $F_{t r}(T)$ is not defined. Thus in this case one has the interval $-\frac{E_{n_{0}}}{\left|S_{n_{0}}\right|}<T<0$ of "forbidden" temperatures. In the particular case $S_{n}=-\frac{E_{n}}{a}, n=1,2, \ldots(a>0)$, this interval is $-a<T<0$ and it represents the tropical limit of the situation discussed in 37 .

Systems with unbounded from below energy spectrum may have lower limit for temperature (see e.g. [38]). In the tropical limit one has $(T>0)$

$$
F_{t r}=-T \sum_{-\infty<n<\infty} \oplus\left(-\frac{F_{n}}{T}\right)=\min \left\{\ldots, F_{-2}, F_{-1}, F_{0}, F_{1}, F_{2}, \ldots\right\}
$$

where $E_{n}<0$ at $n<0$. The tropical sum in 5.2 might diverge due to the negative terms $F_{-n}=E_{-n}-S_{-n} T(n=1,2, \ldots)$. Indeed, let there exist certain $m_{0}>0$ such that all $S_{-m_{0}-k}(k=0,1,2, \ldots)$ are negative, $\left|S_{-m_{0}-k-1}\right|>\left|S_{-m_{0}-k}\right|(k=0,1,2, \ldots)$ 
and $\frac{E_{-m_{0}-k}}{S_{-m_{0}-k}} \leq \frac{E_{-m_{0}-k-1}}{S_{-m_{0}-k-1}}, k=0,1, \ldots$. Then at $T<T_{S-}:=\frac{E_{-m_{0}}}{S_{-m_{0}}}$ one has

$$
\begin{array}{r}
F_{-m_{0}}=\left|S_{-m_{0}}\right| \cdot\left(T-T_{S-}\right)>\left|S_{-m_{0}-1}\right| \cdot\left(T-\frac{E_{-m_{0}-1}}{S_{-m_{0}-1}}\right)>\ldots \\
\ldots>\left|S_{-m_{0}-k}\right| \cdot\left(T-\frac{E_{-m_{0}-k}}{S_{-m_{0}-k}}\right)>\ldots
\end{array}
$$

So the sequence $F_{-m_{0}}, F_{-m_{0}-1}, F_{-m_{0}-2}, \ldots$ is strictly decreasing and it can be unbounded from below at $T<T_{S-}$. In such a case $F_{t r}(T)$ does not exist at $T<T_{S-}$ and $T_{S-}$ is the lower limiting temperature.

Combining the above case and that considered the first in this section, one gets the system with unbounded both from below and above energy spectrum which can stay in equilibrium at the temperature belonging to the finite interval $T_{S-}<T<T_{S+}$ with $T_{S+}=\frac{E_{n_{0}}}{S_{n_{0}}}$ and $T_{S-}=\frac{E_{-m_{0}}}{S_{-m_{0}}}$.

More complicated cases with intervals of allowed (or forbidden) temperatures as well as the behavior of such systems at $T=T_{S}$ will be discussed elsewhere.

Acknowledgments. The second author (B.K.) was partially supported by the PRIN 2010/2011 grant 2010JJ4KBA_003.

\section{References}

[1] G. L. Litvinov and V. P. Maslov, Idempotent mathematics: correspondence principle and applications, Russ. Math. Surveys, 51, (1996), no. 6, 1210-1211.

[2] G. L. Litvinov and V. P. Maslov, The correspondence principle for idempotent calculus and some computer applications, in: Idempotency, Ed. by J. Gunawardena, Cambridge Univ. Press, Cambridge, 1998, pp. 420-443.

[3] G. L. Litvinov, The Maslov dequantization, idempotent and tropical mathematics: a brief introduction, J. Math. Sciences, 140, (2007), 426-444, arXiv:math:GM/0507014.

[4] T. Tokihiro, D. Takahashi, J. Matsukidaira and J. Satsuma, From soliton equations to integrable cellular automata through a limiting procedure, Phys. Rev. Lett., 76 , (1996), 3247-3250.

[5] G. R. W. Quispel, H. W. Capel and J. Scully, Piecewise-linear soliton equations and piecewise linear integrable maps, J. Phys. A, Math. Gen. 34, (2001), 2491-2503.

[6] G. Hatayama, K. Hikami, R. Inoue, A. Kuniba, T. Takagi and T. Tokihiro, The $A_{M}^{(1)}$ automata related to crystals of symmetric tensors, J. Math. Phys., 42, (2001), 274-308. 
[7] A. Dimakis and F. Müller-Hoissen, KP line solitons and Tamari lattices, J. Phys. A: Math. Phys., 44, (2011), 025203 (49 pp.).

[8] R. Inoue, A. Kuniba and T. Takagi, Integrable structure of box-ball systems: crystals, Bethe ansatz, ultradiscretization and tropical geometry, J. Phys. A: Math. Theor., 45, (2012), 073001 (64 pp.).

[9] G. Mikhalkin, Enumerative tropical algebraic geometry, J. Amer. Math. Soc., 18, (2005), 313-377.

[10] I. Itenberg, G. Mikhalkin and E. I. Shustin, Tropical algebraic geometry, Oberwolfach Seminars, vol. 35, Birkhäuser, Basel, 2009.

[11] A. Gathmann and H. Markwig, Kontsevich's formula and the WDVV equations in tropical geometry, Adv. Math., 217, (2008), 537-560.

[12] O. Ya. Viro, On basic concepts of tropical geometry, Proc. Steklov Institute Math., 273, (2011), 252-282, Pleiades Publ. Ltd.

[13] I. Itenberg and G. Mikhalkin, Geometry in tropical limit, Math. Semesterberichte, 59, (2012), 57-73.

[14] G. L. Litvinov and S. N. Sergeev (Eds.), Tropical and Idempotent mathematics, Contemporary Mathematics, vol. 495, AMS, Providence, RI. 2009.

[15] C. Athorne, D. Maclagan and I. Strachan (Eds.), Tropical geometry and integrable systems, Contemporary Mathematics, vol. 580, AMS, 2012.

[16] D. Maclagan and B. Sturmfels, Introduction to Tropical Geometry, Graduate Studies in Mathematics, AMS, Providence, RI, 161, 2015

[17] J. P. Quadrat and the Max-Plus working group, Min-Plus linearity and statistical mechanics Markov Processes and Related Fields, 3, (1997), 565-587.

[18] L. Pachter and B. Sturmfels, Tropical geometry of statistical models, PNAS, 101, no. 46, (2004), 16132-16137.

[19] R. Kenyon, A. Okounkov and S. Sheffield, Dimers and Amoebae, Ann. Math., 163, (2006), 1019-1036.

[20] M. Kapranov, Thermodynamics and the moment map, arXiv:1108.3472, 2011.

[21] M. Marcolli and R. Thorngren, Thermodynamic semirings, J. Non-commutative Geometry, 8, (2014), 337-392.

[22] L. D. Landau and E. M. Lifschitz, Statistical physics, part 1, vol.5, ButterworthHeinemann, 1980.

[23] L. Pauling, The structure and entropy of ice and of other crystals with some randomness of atomic arrangement, J. Am. Chem. Soc., 57, 12, (1935), 2680-2684. 
[24] W. F. Giauque and M. F. Ashley, Molecular Rotation in Ice at 10 K. Free Energy of Formation and Entropy of Water, Phys. Rev., 43, (1933), 81-82.

[25] G. H. Wannier, Antiferromagnetism and the triangular Ising net, Phys. Rev. 79, (1950), 357-364.

[26] R. Moessner and S. L. Sondhi, Ising models of quantum frustration, Phys. Rev. B 63, (2001), 224401.

[27] S. T. Bramwell and M. J. P. Gingras, Spin ice state in frustrated magnetic pyrochlore materials, Science, 294, no. 5546, (2001), 1495-1501.

[28] H. T. Diep, (Ed.), Frustrated spin sytems, World Scientific, Singapore, 2005.

[29] G. C. Lau, et. al., Zero point entropy of stuffed spin ice, Nature, Science, 2, (2006), 249-253.

[30] P. D. Gujrati, Where is the residual entropy of glass hiding? arXiv:0908.1075 (2009).

[31] I. Gutzow and J. W. P. Schmelzer, The third principle of thermodynamics and the zero-point entropy of glasses, J. Non-Crystalline Solids, 355, (2009), 581-594.

[32] S. V. Nemilov, Zero-point entropy of glasses as physical reality, J. Non-Crystalline Solids, 355, (2009), 607-616.

[33] M. J. P. Gingras, Spin ice, in Highly Frustrated Magnetism, (C. Lacroix et. al., Eds), Springer, Berlin, 2011.

[34] B. H. Lavenda and E. Santamato, Thermodynamic criteria governing irreversible processes under the influence of small thermal fluctuations, J. Stat. Phys., 29, (1982), 345-361.

[35] G. M. Bergman, The logarithmic limit-set of an algebraic variety, Trans. Am. Math. Soc., 157, (1971), 459-469.

[36] N. F. Ramsey, Thermodynamics and statistical Mechanics at negative absolute temperatures, Phys. Rev. 103, (1956), 20-28.

[37] Yu. B. Rumer, Negative and limiting temperatures, JEPT, 38, (1960), 1899-1902.

[38] Yu. B. Rumer and M. Sh. Ryvkin, Thermodynamics, Statistical Physics, and Kinetics, MIR Publ., Moscow, Russia, 1980. 